overwhelms emergency medical services personnel and equipment. In limited-resource environments, e.g., low- to middle-income countries (LMCIs), where healthcare systems already are constrained severely, training needs adaptation and adequate response principles need redefinition.

Methods: Several MCIs during a one-year period in Freetown, Sierra Leone were reviewed. Assessment of personnel, supplies, procedures, and infrastructure was applied to $\mathrm{MCI}$ responses in order understand the modifications required for effective training in limited-resources systems. The MCIs included stadium stampedes, multi-vehicle collisions, and several fires.

Results: In 2008, a needs assessment of surgical capacity at 10 government hospitals in Sierra Leone showed that Connaught, the 267-bed referral center in Freetown, suffered interruptions in oxygen and electricity and lacked basic supplies/equipment. The hospital had six operating rooms; but only two functioned. An analysis of a fire indicated that 40 patients were transported by private vehicles and the fire brigade. While surgeons were knowledgeable regarding essential procedures including cricothyroidotomy, tube thoracostomy, and fracture reduction; supplies were scarce. No surgeries were performed within the first six hours of the event. Chest $\mathrm{x}$-rays were not available until the following day; subsequently two chest tubes were placed. Twenty-six patients died (mortality $=65 \%$ ).

Conclusions: Pre-existing MCI training programs do not meet challenges found in caring for victims where resources are severely constrained. Based on the analysis of the Freetown MCIs, a training course taking resource availability into consideration was developed. The prerequisites of implementing command/control/coordination/communication (C4) remain identical in systems with varying resources. Course curriculum and pertinent adaptations of MCI response training should focus on: (1) resource deployment; (2) evaluating C4 operations; (3) application of skills/professionalism; (4) casualty management; (5) evacuation through traffic; and (6) ascertaining Ministry of Health/local government capacity for handling mass casualties. Finally, LMIC governments should prepare by conducting drills and stockpiling supplies needed for adequate emergency response.

Keywords: developing countries; disaster; limited resources; masscasualty incident; Sierra Leone

Prebosp Disaster Med

\section{Time Standing Still: Adding Realism to Tabletop Exercises John Kimball}

Chief of Special Operations (ret), Fairfax County Fire and Rescue Department, Virginia USA

One of the greatest challenges in preparing for mass-casualty incidents is adding the realism of time-driven decisionmaking while fostering the training message. A close second to this challenge is minimizing stress and pressure and avowing bruised egos. While practicing decision making under the pressure of time is a necessity of incident preparation, real life never gives us the opportunity during an incident to call "time out" before continuing to the next phase.

A mechanism to achieve this sense of urgency is a tabletop exercise in which the incident is parceled into time blocks and the incident management team is divided by function and/or location. Examples of functional groups are incident site, communications, hospital network, and mutual-aid organizations. The incident scenario is introduced in three phases: (1) initial response or stabilization; (2) continued response and operations; and (3) demobilization.

After the real-time period of exercise play, incident time is suspended and the functional groups discuss within their groups the actions taken, what they might have done differently, and what needs to be completed. After the discussion, the functional groups report to the group as a whole. At any point, discrete skills can be reinforced by training reminders. Among these are the development of incident objectives, adjusting assignments, and site management.

This exercise method can be employed to enhance and refine Incident Action Planning, transition of command, and other incident management skills, as well as validating plans and procedures. The method also may be employed to pre-plan emergency resource requirements. In this session, the participants will employ this methodology and focus on the development of the Incident Action Plan and the transition from response to demobilization or longterm operations.

Keywords: functional group; management; mass-casualty incident; planning; tabletop exercise

Prehosp Disaster Med

Notification and Communication: Critical Initial Steps in Mass-Casualty Incident Drills

Carlton Barnett, MD; Jeffry Kashuk, MD;

Ernest E. Moore, MD; Christopher Colwell, $M D$;

Jeffrey Johnson, MD; Walter Biff, $M D$;

Clay C. Berlew, MD; Aaron Brody, MD;

Allison Sabel, $M D, P b D$

Denver Health Medical Center and University of Colorado, Denver Health Sciences Center, Denver, Colorado USA

Background: The global terrorism epidemic and recent disasters caused by natural hazards have underscored the sudden loss of standard methods of communication, which may seriously compromise a hospital's ability to implement the mass-casualty incident (MCI) plan. After initiating hospital-wide drills based on the Israeli approach, it was theorized that notification and communication could be improved with an MCI-specific notification system and a dedicated back-up radio plan.

Methods: Hospital staff completed post-exercise questionnaires evaluating the Regional Level-1 Trauma Center's most recent $\mathrm{MCI}$ drill using Likert-scaled items from 1-10 (worst to best). Participants were instructed to answer 26 items that applied to their experience. Notification and communication issues were assessed specifically in the most recent drill after the implementation of a computerized, one-touch notification system that simultaneously notified staff via beeper, e-mail, and cellular telephone, as well as overhead paging of the occurrence of the drill. Leadership personnel evaluated communication through the use of designated frequency radios distributed to key areas (triage, red area, operating room) of the drill. Responses were compared using the median and interquartile range (IQR). 
Results: A total of 117 staff participated in the drill. The median notification score was $6.5(\mathrm{IQR}=3.5-9.0)$. The effectiveness and timeliness of the mass-notification system tied for the best notification categories by the staff while $39 \%$ felt the quality of the email message only rated a "1". Radio communication received only moderate scores.

Conclusions: Mass notification of personnel with onetouch notification permits the maximum staff response to a MCI. Pilot tests should be performed to determine if staff comprehends the message.

Reliable back-up communication systems should be readily available and tested during drills. Marginal scores for these systems suggest hospital personnel inexperience that would be improved with additional drills.

Keywords: communication; drill; mass-casualty incident; notification

Prehosp Disaster Med

Live Victim Volunteers Enhance Performance Improvement in Mass-Casualty Incident Drills: Listen to the Patient! E.E. Moore; J.L. Kashuk; C. Colwell; C. Barnett; C.C. Burlew; J.L Johnson; W.L. Biffl; A. Brody, A. Sabel

Denver Health Medical Center and Unjversity of Colorado Health Sciences Center, Denver, Colorado USA

Background: Despite growing regulatory requirements for the implementation of a hospital-wide mass-casualty incident (MCI) plan, most trauma centers lack an in-hospital protocol, and little data are available to guide the development of drills. This plan is tailored after the Israeli approach. The purpose of this study was to determine whether live volunteer victims, in lieu of dolls, would provide important data and improve drills.

Methods: Live victim volunteers and hospital staff completed post-exercise questionnaires that evaluated the Regional, Level-1 Trauma Center's most recent MCI drill using Likert-scaled items from 1-10 (worst to best) Participants were instructed to answer any of the 26 items that applied to their experience during the drill. Responses to each question were compared using the median, interquartile range, and Mann-Whitney $U$-test. Statistical significance was set at $p<0.05$.

Results: Twenty-nine live victim voluteers and 117 staff members participated. In $81 \%$ of responses, LVV ratings were higher than those of the hospital staff; in particular, security was valued significantly higher. Of note, digital camera profiling in admissions was rated the highest.

Conclusions: These results emphasize that while more difficult to coordinate logistically, the use of live victim volunteers provide important insights into $\mathrm{MCI}$ drill conduct for performance improvement. For hospital wide drills, live victim volunteers should be used instead of manikkins.

Keywords: drill; live victim volunteer; mass-casualty incident;

performance; victim; volunteer

Prebosp Disaster Med
Medical Simulation as a Training Tool for the Emergency Department Reinforcing Medical Staff in MassCasualty Events

G. Hyams, ${ }^{1}$ L. Otits, ${ }^{2}$ H. Baruch, ${ }^{3}$ M. Michaelson, ${ }^{1}$

M. Barzelay; ${ }^{1}$ S. Kradsbtein; ${ }^{1}$ E. Tal Or ${ }^{1}$

1. Trauma Unit, Rambam Health Care Campus, Haifa, Israel

2. Nursing Administration, Rambam Health Care Campus, Haifa, Israel

3. Emergency Department, Rambam Health Care Campus, Haifa, Israel

Introduction: A mass-casualty event (MCE) is defined as a situation with more casualties than the system can manage, demanding more medical professionals than available. As a solution, the emergency department (ED) recruits staff from the surgical division and the intensive care unit (ICU). In order to train this staff a training program based on simulation was developed and executed.

Methods: An instructional program was developed for the medical staff. The program includes lectures about MCEs with computerized drills, followed by videotaped simulation scenarios of injured people (adult and child). The training is provided to a group of 12 nurses and five physicians assembled in the ED. The training begins with a short lecture about mass-casualty trauma life support and team work, followed by simulations, using the same space and equipment that would be used in a real event. The simulation occurs in a noisy environment with an increased presence of medical staff. At the end of the simulation, the participants review the film and debrief it.

Results: During the last four years, 191 nurses and 61 physicians were trained. The trainees reported that the goals achieved (4.76 out of 5), the scenarios were appropriate and reflected real scenarios of trauma (4.53). The simulations added new knowledge (4.16) and contributed to better performance (4.16). The combination between the theoretical and practical knowledge contributed to their confidence.

Conclusions: During the $2^{\text {nd }}$ Lebanon War, the trainees had the opportunity to use this knowledge in real-time events. It is highly recommended to train teams in the environment and with the equipments and records that will be used in a real MCE. The simulations mimic the real situation as much as possible. An important aspect of each simulation is the videotaping and the debriefing. The trainees appreciated this plan that enhanced their skills, knowledge, abilities, and appointed role. Training by simulations provides a good method in light of a limited budget and shortage of personnel. In times of a limited budget, the training should be goal-focused and short in duration.

Keywords: emergency department; mass-casualty incident; medical; staff; simulation; training

Prebosp Disaster Med 